Dagmar Schiek

\title{
"Vereinbarkeit von Beruf und Familie« - vereinbar mit der arbeitsmarktlichen Gleichstellung von Frauen?
}

Am I. September 1994 ist das Zweite Gleichberechtigungsgesetz in Kraft getreten ${ }^{\text {. }}$ Erklärtes Ziel des Gesetzesvorhabens ist es, "zusätzliche gesetzliche Regelungen (...) zur besseren Vereinbarkeit von Familie und Beruf « zu schaffen ${ }^{2}$. Auch die acht neueren Landesgleichstellungsgesetze ${ }^{3}$ enthalten Regelungen zur Vereinbarkeit von Beruf und Familie. Nur die Quotenvorschriften der beiden ältesten Frauenfördergesetze der Länder Nordrhein-Westfalen und Saarland 4 verzichten auf solches Beiwerk. Regelungen zur Förderung der besseren Vereinbarkeit von Familie und Beruf gelten manchem als wesentlich erfolgversprechenderes Konzept der Herstellung von Chancengleichheit zwischen Frauen und Männern als die Quotierungs und werden auch von Kritikern der Quote wärmstens empfohlen ${ }^{6}$. Das Thema der arbeitsrechtlichen Abteilung des 60. Juristentages "Welche Maßnahmen empfehlen sich, um die Vereinbarkeit von Berufstätigkeit und Familie zu verbessern? « wurde denn auch von beiden Gutachtern als ausgesprochenes Frauenthema verstanden. Soviel Einigkeit provoziert die Fragestellung, ob Vereinbarkeit, wie sie das gegenwärtige Arbeitsund Dienstrecht - auch nach der Änderung durch das Zweite Gleichberechtigungsgesetz - versteht, überhaupt etwas mit der Gleichstellung von Frauen und Männern am Arbeitsmarkt zu tun hat.

\section{A. „Vereinbarkeit von Beruf und Familie im geltenden Recht}

Die kontinuierliche Verantwortung für die alltägliche Betreuung von Kindern liegt von wenigen Ausnahmefällen abgesehen - bei Frauen. »Vereinbarkeitsregeln« sind deshalb vorrangig Frauenrecht. In der modischen Diskussion um »Vereinbarkeit« geht dieser Fakt häufig in geschlechtsneutralen Formulierungen unter. Geschlechtsspezifische Formulierungen aber, die an die bemerkenswert veränderungsresistente Realität anknüpfen, scheinen den Anspruch auf Veränderung dieser einseitig Frauen belastenden Arbeitsteilung aufzugeben - ein unlösbares Dilemma. Als unbefriedigender Kompromiß wird im folgenden von aktiven Eltern gesprochen, die zu 99,9\% Mütter sind - die Leserin und der Leser möge jeweils über das Wort »aktiv« stolpern

I Vom 21. 4. I994, BGBI. I, 1406

2 Vgl. Gesetzentwurf der Bundesregierung vom 21.7.1993, BT-Drs. 12/5468, unter A. Zielsetzung; Abg. Nolte (CDU), 222. Sitzung des Bundestages am 21.4.1994 (Zweite und Dritte Lesung des 2. GleibG), Stenographischer Bericht S. 19153 (B); Abg. Böhmer (CDU), ebenda, S. 19159 (D).

3 In der Reihenfolge des Inkrafttretens: Landesgleichstellungsgesetz Bremen vom 29. I I. 1990 (BremGVBI. S. 433); Berliner Landesgleichstellungsgesetz vom 31. 12. 1990 (BlnGVB1. 1991, 8), geändert durch Gesetz vom I 3. 4. 1993 (BlnGVBl. I 84); Hamburgisches Gleichstellungsgesetz vom 19.3. I 99 I (HambGVBl. 75, in Kraft seit 1.4.1992); Frauenfordergesetz des Landes Sachsen-Anhalt vom 27.6.1993 (GVBI. 762); Hessisches Gleichberechtigungsgesetz vom 13. I 2. 1993 (HessGVBl. 729); Gleichstellungsgesetz Mecklenburg-Vorpommern vom 14.3.1994 (MV GVBl. 343); Sächsisches Frauenfördergesetz vom 31.3. 1994 (SähsGVBI. 684); Niedersächsisches Gleichberechtigungsgesetz vom 16.6. 1994 (NdsGVBl. 246); Brandenburgisches Landesgleichstellungsgesetz vom 8.7.1994 (GVBI. Brdbg Teil I, 254).

4 Saarländisches Gesetz zur Förderung von Frauen und zur Änderung sonstiger dienstrechtlicher Vorschriften vom 6.7.1989, ABI. Saarl. S. 977, nordrhein-westfalisches Frauenfördergesetz vom 31. 10. 1989, GV. NW. S. 567 .

5 Battis, Eisenhard, ZRP 1994, 18 (20).

6 Ladeur, ZRP 1993, 47; Sachs, NJW 1988, 553 ( 556 liSp). 
und sich an die überwältigende Realität verantwortungsloser männlicher Elternschaft erinnern.

Als Regelungen zur "Vereinbarkeit« werden meist solche arbeitsrechtlichen Vorschriften bezeichnet, die aktiven Eltern eine relativ abgesicherte Unterbrechung der Erwerbstätigkeit, familienbedingte Teilzeitarbeit und/oder flexible Arbeitszeiten ermöglichen. Hinzu kommen Regelungen, die während einer Unterbrechungszeit eine Einkommenssicherung gewährleisten sowie Vorschriften der Jugendhilfe, die die auBerfamiliäre Betreuung von Kindern während der Arbeitszeit aktiver Eltern gewährleisten.

\section{Abgesicherte Unterbrechung der Erwerbstätigkeit}

Am besten abgesichert ist die Unterbrechung der Erwerbstätigkeit der Mutter unmittelbar nach der Geburt: während der vierzehnwöchigen mutterschutzrechtlichen Schutzfristen ( $\$ 3$ Abs. I und $\$ 6$ Abs. I MuSchG) besteht Arbeitsplatzschutz ( $\$ 9$ MuSchG) und Entgeltschutz. Die Gleichstellung von Beamtinnen und Arbeitnehmerinnen gewährleisten die Mutterschutzverordnungen des Bundes ${ }^{7}$ und der Länder.

Im Anschluß an die Schutzfristen ermöglicht der Erziehungsurlaub nach $\$$ Is BErzGG weitere Unterbrechungszeiten. Anspruchsberechtigt sind seit $1992^{8}$ neben den sorgeberechtigten Personen auch der nichteheliche oder geschiedene Vater $(\$ \mathbf{I}$ Abs. 3 Nr. 3 BErzGG) und in Härtefällen weitere Verwandte ( $\$$ I Abs. 7 BErzGG). Das Kündigungsverbot nach $\$ 18$ BErzGG entspricht nach dem gesetzlichen Wortlaut $\$ 9$ MuSchG. Allerdings hat die Bundesregierung hier einheitliche Verwaltungsvorschriften erlassen?, die diesen Kündigungsschutz bei Existenzgefährdung des Betriebes und bei Kleinbetrieben auflockern. Kündigungen im Zusammenhang mit dem Erziehungsurlaub wurden nach dem ersten und einzigen Erfahrungsbericht der Bundesregierung in $0,14 \%$ der Fälle ausgesprochen ${ }^{10}$. Seit dem I. I. 1992 besteht Kündigungsschutz ab der sechsten Woche vor Antritt des Erziehungsurlaubs, so daß beurlaubungswillige Väter oder andere Verwandte während der Ankündigungsfrist von vier Wochen ( $\$$ I 6 Abs. I S. I BErzGG) nicht mehr um den Arbeitsplatz bangen müssen. Ein Kündigungsschutz im Anschluß an den Erziehungsurlaub - wie nach $\$ 9$ MuSchG - besteht nicht, jedoch ist eine Kündigung aus Anlaß des Erziehungsurlaubs wegen Verstoßes gegen das Benachteiligungsverbot des $\$ 6$ I 2 a BGB unwirksam $^{11}$.

Für Beschäftigte der Privatwirtschaft werden die Möglichkeiten der »familienbedingten « Unterbrechung der Erwerbstätigkeit durch einige betriebliche und tarifliche Regelungen ergänzz ${ }^{12}$. Vorrangig Großbetriebe ermöglichen eine verlängerte „Familienpause«. Bei diesen betrieblichen "Familienpausen« ist der Arbeitsplatzschutz im Vergleich zum Bundeserziehungsgeldgesetz unvollständig.

Aktive beamtete Eltern können im Anschluß an den Erziehungsurlaub zur Betreuung minderjähriger Kinder Sonderurlaub von drei Jahren mit der Möglichkeit der

7 BGBl. I 1991, I 26.

8 Zur Neuregelung Zmarzlik, FuR 1992, S. 313.

9 Allgemeine Verwaltungsvorschriften zum Kundigungsschutz bei Erziehungsurlaub $\$ 18$ Abs. I S. 3 BErzGG vom 2. 1. 1986, BAnz Nr. I/1986.

10 BT-Drs. I 1/8517. 1987: 376 Kündigungen bei 267338 Erziehungsurlaubsfällen; 1988: 363 Kundigungen bei 283404 Erziehungsurlaubsfallen.

I I Zmarzluk, FuR 1992, S. 313 .

12 Vgl. hierzu ausfuhrlich Colneric, RdA 1994, S.65 und Brumlop, Homung, Betriebliche Frauenförderung - Aufbrechen von Arbeitsmarktbarrieren oder Verfestigung traditioneller Rollenmuster? 1993 (SAMF Arbeitspapier). 
Verlängerung auf bis zu fünfzehn Jahre beanspruchen ( $\$ 48$ a BRRG, $\$ 79$ a BBG und entsprechende Regelungen der Landesbeamtengesetze). Auf Gewährung des Urlaubs bestand schon nach altem Recht ein Anspruch, wenn die erforderliche Abwägung der Interessen der Beamtin und der Dienststelle keine andere Entscheidung erlaubte. Durch das Zweite Gleichberechtigungsgesetz wurden diese Rechte graduell verbessert: Nach $\$ 48$ a BRRG n.F. und dem entsprechend geänderten $\$ 79$ a BBG haben Beamtinnen nunmehr einen Anspruch auf Beurlaubung, wenn dem nicht zwingende dienstliche Belange entgegenstehen. Der Arbeitsplatzschutz nach der Beurlaubung ist absolut, allerdings hat der Dienstherr das Recht zur Versetzung an einen anderen Dienstort. Für privatvertraglich Beschäftigte des öffentlichen Dienstes bildet $\$ 50$ Abs. 2 BAT das Korrelat zu $\$ 48$ a BRRG. Nach der Rechtsprechung darf ein Antrag nach $\$$ s० Abs. 2 BAT nicht abgelehnt werden, wenn die Beurlaubung zur Betreuung eines Kleinkindes erforderlich ist und keine berechtigten dienstlichen Interessen entgegenstehen ${ }^{13}$. Der Anspruch reicht allerdings nicht so weit wie der beamtenrechtliche Beurlaubungsanspruch ${ }^{14}$. Die Landesgleichstellungsgesetze der Länder Berlin, Bremen, Hamburg, Hessen und Niedersachsen dehnen - weitergehend als das Zweite Gleichberechtigungsgesetz - die beamtenrechtlichen Beurlaubungsmöglichkeiten auf privatvertraglich Beschäftigte aus.

\section{Familienbedingte Teilzeitarbeit}

Ein arbeitsrechtlicher Anspruch auf Berücksichtigung der Wünsche aktiver Eltern nach vorübergehender Reduzierung der Arbeitszeit besteht nicht. Wird die Arbeitszeit reduziert, haben Beschäftigte nach überwiegender Meinung keinen Anspruch auf Aufstockung der Arbeitszeit zu einem späteren Zeitpunkt ${ }^{\text {is }}$. Inwieweit etwas anderes gilt, wenn der Teilzeitarbeit bzw. der Aufstockung keine betrieblichen Belange entgegenstehen, die die familiären Interessen der oder des Beschäftigten aufwiegen können, ist noch nicht geklärt ${ }^{16}$. Einige Betriebsvereinbarungen zur "Familienförderung « sehen sowohl die Teilzeitbeschäftigung als auch die Rückkehr zur Vollzeittätigkeit als Möglichkeit vor.

Aktive beamtete Eltern haben neben dem Anspruch auf familienbedingte Beurlaubung einen Anspruch auf familienbedingte Reduzierung der Arbeitszeit. Die ErmäBigung der Arbeitszeit darf - auch in Zeiten knapper Kassen - nur freiwillig erfolgen. Eine Ermäßigung aufgrund eines Antrages, den eine Bewerberin oder ein Bewerber auf Veranlassung der Dienststelle stellt, um überhaupt angestellt $\mathrm{zu}$ werden, ist rechtswidrig, wenn auch nicht nichtig ${ }^{17}$.

Sonstige aktive Eltern im öffentlichen Dienst haben in bezug auf Teilzeitarbeit die-

13 BAG 12. I. 1989, EzBAT Nr. $\$$ so BAT Unbezahlter Sonderurlaub, zuvor ArbG Saarbricken, 20. I1. 1987, EzBAT Nr.4 zu so BAT Unbezahlter Sonderurlaub, ArbG Wetzlar, 8. 5. 198 s, NZA 198 s, 536.

14 So hat das Arbeitsgericht Mainz entschieden, der Wunsch einer Halbtagskraft, ihre beiden schulpflichtigen Kinder ganztags zu betreuen, sei kein wichtiger Grund im Sinne des $\$$ so Abs. 2 BAT; vielmehr sei die Beteiligung des Vaters, der als Lehrer jedenfalls nachmıttags seine Zeit frei éinteilen könne, an der Kinderbetreuung zumutbar; Urt. v. 16.8.1990, EzBAT Nr. 6 zu $\$$ so Abs. 2 BAT Unbezahlter Sonderurlaub.

is Allg. Meinung, s. nur Schuren, Munchener Handbuch, $\$ 158 \mathrm{Rz} 2$ bis $4 \mathrm{~m}$.w. N.

I6 Allerdings entschied das ArbG Hamburg, daß die Kündigung einer alleinerziehenden Mutter wegen schichtplanwidrigem Erscheinen zur Frühschicht statt zur Spätschicht sozialwidrig ist, wenn die Frau ohne Beeinträchtigung betrieblicher Belange außerhalb des Schichtbetriebes in Teilzeit beschaftigt werden kann und hiermit einverstanden ist (15.1 $1,198_{3}$, Streit 1984,95$)$.

17 BVerwG 4. 3. I $992-, 2$ Bl 8.92-RiA 1993, 96. 
selbe Position wie in der Privatwirtschaft ${ }^{18}$. Einige Landesgleichstellungsgesetze ${ }^{19}$ geben allen Beschäftigten des öffentlichen Dienstes das Recht auf familienbedingte Teilzeit mit Rückkehrmöglichkeit zur Vollzeitbeschäftigung. Darüber hinaus fordern die acht neueren Landesgleichstellungsgesetze und das Frauenfördergesetz für die Bundesverwaltung für alle Statusgruppen, daß genügend Teilzeitarbeit angeboten werden soll, auch in Führungspositionen. In einigen Bundesländern sollen Dienststellen familiengerechte Arbeitszeiten anbieten (Berlin, Bremen, Hessen, Mecklenburg-Vorpommern, Sachsen), in Bremen ausdrücklich auch für Vollzeitbeschäftigte.

\section{Eigenständige Existenzsicherung aktiver Eltern und ihrer Kinder}

Die Einkommenssicherung von Mutter und Kind ist am besten im Mutterschutz gewährleistet: Das Nettoeinkommen bleibt erhalten, da der Arbeitgeber den $\mathrm{Zu}$ schuß der Krankenkasse von 25 Mark täglich auf dessen Höhe aufstocken muß. Allerdings muß die Mutter eventuell Nachteile in der Rentenversicherung hinnehmen: Die Mutterschutzfrist wird unabhängig vom individuellen Einkommen nur mit 0,66 Entgeltpunkten belegt ( $\$ \mathbb{S}_{3}$ Abs. 1, 56, $\left.249 \mathrm{SGB} \mathrm{VI}\right)$. Zwar wirken sich die 14 Wochen nicht erheblich auf die Rentenhöhe aus, aber es erscheint doch seltsam, daß mit dem mutterschutzrechtlichen Beschäftigungsverbot ein Rentenverzicht verordnet wird. Nach dem BErzGG wird kein Entgeltschutz gewährt; die 600 Mark Erziehungsgeld - seit 1993 für die gesamte Laufzeit einkommensabhängig ${ }^{20}$ - müssen als Prämie für das Ausscheiden aus dem Erwerbsleben betrachtet werden. Da das Arbeitsverhältnis während des Erziehungsurlaubs nur ruht, sind leistungsunabhängige Entgeltbestandteile - wie etwa die Jahressonderzahlung - weiterzugewähren, solange Tarif- oder Arbeitsvertrag nichts anderes bestimmen. ${ }^{21}$ Eine Existenzsicherung durch Erwerbstätigkeit während des Erziehungsurlaubs ist nur eingeschränkt möglich: Gearbeitet werden darf nicht mehr als 19 Stunden ( $\$ 2$ BErzGG) und nur mit Zustimmung des Arbeitgebers ( $\$$ is Abs. \$). An den Erziehungsurlaub anschlieBende "Elternfreistellungen " bieten gar keine finanzielle Sicherung. Dennoch wird die "Familienpause" in Betriebsvereinbarungen häufig nur unter der Bedingung gewährt, daß keine anderweitige Erwerbstätigkeit aufgenommen wird; das gilt per Gesetz auch für die beamtenrechtliche Beurlaubung. Bei familienbedingter Teilzeitarbeit werden finanzielle Aspekte vollkommen vernachlässigt; sie wird ausschließlich mit proportionaler Einkommensreduktion angeboten und sichert deshalb in den seltensten Fällen eine eigenständige Existenz. Dies gilt auch unter Berücksichtigung eventueller Kindesunterhaltszahlungen. Interessanterweise steht bei arbeitsmarktbedingter Teilzeit, die ja auch Männern auferlegt wird/werden soll, die Frage nach Lohnersatzleistungen oder der Gewährleistung der Existenzsicherung im Vordergrund.

18 Nur in dem Ausnahmefall, daß ein Arbeitgeber beamtenrechtliche Vorschriften pauschal auf Arbeitsverhältnisse anwendet, kann ein Anspruch auf Teilzeitbescháftigung entsprechend den beamtenrechtlichen Regelungen - d. h. mit Arbeitsplatzgarantie - entstehen (BAG 20. 1. 1992, S AZR 266/90-, EzBAT $\$$ is BAT Herabsetzung der Wochenarbeitszeit Nr. 2).

19 Bremen, Hamburg, Hessen, Niedersachsen.

20 Fur die ersten sechs Monate wird nur ein Einkommen uber 75000 Mark jährlich (Verheiratete: 100000 Mark) angerechnet. Anschließend bleibt Einkommen von 23700 Mark jährlich (Verheiratete 29400 Mark) anrechnungsfrei, das diesen Betrag übersteigende Einkommen wird zu $40 \%$ angerechnet. Die Beträge verstehen sich jeweils zuzuglich 4200 Mark jährlich fur jedes Kind ( $\$$, BErzGG).

21 BAG DB I99 1, 446; LAG Duisseldorf 29. 1 1. 1990, DB 1991, 81 3. Nur aufgrund einer eindeutigen tariflichen Regelung kann die Jahressonderzahlung für Monate des Erziehungsurlaubs gekürzt werden; entsprechende Regelungen für den Wehrdienst und andere Urlaubstatbestände sind nicht übertragbar. Es genuigt, wenn die Quotelung neutral fur Zeiten des Ruhens des Erwerbsarbeitsverhältnisses aufgrund Vereinbarung oder Gesetzes vorgesehen ist (BAG AP Nr.7 zu $\$$ is BErzGG). 
Bei den Mutterschutzfristen erscheint ein Nachteilsausgleich weitgehend entbehrlich; sie sind zu kurz, um ernsthafte Dequalifizierungsprozesse einzuleiten. Bei der Rückkehr aus dem Erziehungsurlaub fehlt in der Privatwirtschaft ein Schutz gegen Rückstufung oder Zuweisung eines anderen Arbeitsplatzes - etwa vergleichbar $₫ 6$ ArbPISchG, wonach Wehrpflichtigen aus der Ableistung des Wehrdienstes kein Nachteil entstehen darf. Auch ohne ausdrückliche Regelung besteht allerdings Anspruch auf einen gleichwertigen Arbeitsplatz ${ }^{22}$. Einen gewissen Nachteilsschutz gewährleistet darüber hinaus das gesetzlich angeordnete Ruhen des Arbeitsverhältnisses ${ }^{23}$. Soweit ein »Bewährungsaufstieg « in eine höhere Tarifgruppe nach Ablauf einer bestimmten Beschäftigungszeit in Anerkennung der Betriebstreue ${ }^{24}$ oder nach beanstandungsfreier Arbeitsleistung während dieser Zeit ${ }^{25}$ vorgesehen ist, sind Zeiten des Erziehungsurlaubs Zeiten aktiver Erwerbstätigkeit gleichzustellen. Die Ungleichbehandlung von Erziehungsurlaub und Zeiten aktiver Erwerbstätigkeit oder des Wehrdienstes ist jedoch gesetzlich nicht verboten. Ob Tarifnormen, die Wehrdienst als aufstiegsrelevant bewerten, Erziehungsurlaub aber nicht, als mittelbare Diskriminierung rechtswidrig sind, ist noch nicht entschieden ${ }^{26}$. Bei über den Erziehungsurlaub hinausgehenden betrieblichen "Familienpausen " ist der Nachteilsschutz in der Regel noch schwächer ausgebaut. Einige von ihnen sehen Qualifizierungspflichten oder auch -möglichkeiten vor, wodurch die Dequalifikation begrenzt wird. Im Beamtenrecht ist der statusorientierte Nachteilsschutz bei familienbedingten Unterbrechungen recht komfortabel ausgestaltet. Grundlage ist der nur Frauen begünstigende $₫ 125$ b Abs. 1 BRRG, wonach bei der Prüfung für die fachlichen Eignungsvoraussetzungen für die Einstellung in den öffentlichen Dienst bei einer Bewerberin, die sich innerhalb von zwei Jahren nach der Geburt ihres Kindes beworben hat, zu ihren Gunsten auf den Zeitpunkt abzustellen ist, zu dem sie sich ohne die Geburt ihres Kindes hätte bewerben können ${ }^{27}$. Nach $\$$ I2 BLV und den entsprechenden Laufbahnvorschriften der Länder gelten Zeiten des Erziehungsurlaubs oder einer Beurlaubung nach $₫ 79$ a BBG als Dienstzeiten, die Voraussetzung einer Beförderung sind, allerdings nur für ein Jahr pro Kind und insgesamt - auch bei mehr als zwei Kindern - für höchstens zwei Jahre anzurechnen. Auch während einer familienbedingten Beurlaubung kann befördert werden.

Nach dem Frauenfördergesetz des Bundes darf sich für Bundesbedienstete die Beurlaubung künftig nicht mehr »nachteilig auf den bereits erreichten Platz in einer Beförderungsreihenfolge auswirken « ( $\$ 12$ Abs. 4). Nach $₫ 28$ BBG und den entspre-

22 Metsel, Sowka, Rz 32 a zu $\$ 15$ BErzGG; Zmarzlik, Zipperer, Viethen, Rz, is zu $\$ 18$ BErzGG.

23 BAG 10. 2. 1993, AP Nr. 7 zu $\ 15$ BErzGG.

24 BAG 21. 10. 1992, AP Nr. 1 zu \I TVG - Tarifverträge - Milch- und Kasewirtschaft.

$25 \$ 23$ a BAT - LAG Saarland, 26.8. 1992, Streit 1993, 60; hierzu auch Mauer, NZA r 991, S. 501; s. a. BAG 15. 2. 1994, ArbuR 1994, 1010 zur Wirksamkeit einer Regelung in der Unterstützungskasse des DGB nach der Zeiten des Erziehungsurlaubs nicht betriebsrentensteigernd wirken.

26 Ausdrücklich offengelassen in BAG AP Nr. I zu $\$$ I TVG-Tarifvertrage-Milch- und Käsewirtschaft, Bl 4; für mittelbare Diskriminierung in einem solchen Fall Buglass, Hetlmann, ArbuR r992, 353.

27 Diese Vorschrift wird durch $\$ 10$ BLV und die entsprechenden Laufbahnvorschriften der Länder konkretisiert: Hat sich die Einstellung wegen einer ununterbrochenen Betreuung eines in häuslicher Gemeinschaft lebenden Kindes unter 18 Jahren verzögert, darf die Anstellung nach Erwerb der Laufbahnbefähigung nicht über den Zeitpunkt hinausgeschoben werden, zu dem die Betroffene ohne die Verzógerung zur Anstellung angestanden hätte, sofern die Bewerbung innerhalb von sechs Monaten nach Beendigung der Kinderbetreuung oder nach Beendigung der im Anschluß an die Kinderbetreuung begonnenen vorgeschriebenen Ausbildung gefuhrt hat. Entsprechendes gilt für einen Beamten, der tatsächlich beurlaubt war. Pflegezeiten sind gleichgestellt - allerdings zugunsten von Frauen und Mannern (letzteres durch Änderung der BLV im Vorfeld des Zweiten Gleichberechtigungsgesetzes). 
chenden landesgesetzlichen Vorschriften wird der Beginn des Besoldungsdienstalters für Zeiten einer Kinderbetreuung von bis zu drei Jahren pro Kind nicht hinausgeschoben. Mit dem Zweiten Gleichberechtigungsgesetz wird die Begünstigung des $\$ 125$ b BRRG auf die Betreuung Pflegebedürftiger ausgedehnt-dies für Frauen und Männer gleichermaßen. Benachteiligungen bei der beruflichen Entwicklung infolge von familienbedingten Beurlaubungen sind nicht grenzenlos zulässig. So hielt der Hessische Verwaltungsgerichtshof die vollkommene Vernachlässigung eines familienbedingten Sonderurlaubs von insgesamt sechs Jahren zur Betreuung zweier Kinder bei der Dienstaltersbewertung für ermessensfehlerhaft. Entsprechend einem ministeriellen Erlaß hätte pro Kind ein Jahr des Sonderurlaubs voll und der Rest zu fünfzig Prozent berücksichtigt werden müssen ${ }^{28}$. Auf der Linie dieser Gerichtsentscheidungen bestimmen die neun neueren Landesgleichstellungsgesetze sowie das Frauenfördergesetz des Bundes ( $\$ 7$ Abs. 2), daß Erfahrungen in der »Familienarbeit « bei Beurteilungen positiv zu berücksichtigen sind. Um den drohenden Qualifikationsverlusten bei Beurlaubungen vorzubeugen, sehen die neun neueren Landesgleichstellungsgesetze vor, daß beurlaubte Beschäftigte an Fortbildungen teilnehmen können, nach dem Bremischen, Hamburgischen und Hessischen Gesetz sind Beurlaubten außerdem kurzfristige Vertretungen sowie Wiedereinstiegsfortbildungen anzubieten. Nach dem Frauenfördergesetz des Bundes (Artikel I des Zweiten Gleichberechtigungsgesetzes) sollen - wie auch nach dem sächsischen Frauenfördergesetz - Beurlaubten Fortbildungen unter Erstattung der Reisekosten offenstehen ( $($ I r). Dies gilt für alle Beschäftigtengruppen gleichermaßen.

\section{Teilzeit}

Rechtlichen Benachteiligungen aufgrund von Teilzeitbeschäftigung, die vor allem in Tarifverträgen normiert sind, hat die arbeitsgerichtliche Rechtsprechung in den letzten Jahren zum Teil die Anerkennung verweigert. Grundlage waren das allgemeine Benachteiligungsverbot des $\ 2$ BeschFG und häufiger das Verbot mittelbarer Diskriminierung 29 . Die ausdrücklichen Gleichbehandlungsgebote des Hamburgischen bzw. Hessischen Landesgleichstellungsgesetzes für Teilzeitbeschäftigte gehen nicht darüber hinaus. Das Sächsische Frauenfördergesetz und das Frauenfördergesetz für die Bundesverwaltung schreiben gleiche Fortbildungsmöglichkeiten für Teilzeitbeschäftigte vor, bleiben aber ansonsten hinter dem Verbot der mittelbaren Diskriminierung zurück.

Folgende für Teilzeitbeschäftigte ungünstige Regelungen fielen den beiden Benachteiligungsverboten bereits zum $\mathrm{Opfer}^{3 \circ}$ : Ausschluß von Teilzeitbeschäftigten aus der betrieblichen Altersversorgung ${ }^{31}$, Ausschluß vom Übergangsgeld nach dem $\mathrm{BAT}^{32}$, Ausschluß von Teilzeitbeschäftigten von der gesetzlichen Lohnfortzahlung ${ }^{33}$, Ausschluß von bestimmten Zulagen oder anderen tariflichen Leistungen ${ }^{34}$, Verdoppelung

\footnotetext{
28 HessVGH 5. 4. 1991, I TG 245/91 -, ArbuR 1992, S. 381 m. Anm. Buglass, Hetlmann, ebd. S. 393.

29 Ausführlich Pfarr, Bertelsmann, Diskriminierung im Erwerbsleben, 1989, S. I I sowie Schüren, Munchener Handbuch, $\$$ i $\$ 7$ Rz 89-108.

30 Knapper Überblick über die aktuelle Rechtsprechung: Lıpke, AuA S. 1994, 8, sowie Goergens, AiB 1993 , S. 56.

31 BAG I 4. 3. $1989-3$ AZR 490/87-, NJW 1990, 68 (BILKA, dazu EuGH I 3. 5. 1986, C- 170/84, STREIT

1986, 126), BAG 20.11.1990-3 AZR 613/89-, NZA 1991, 635.

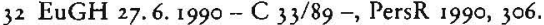

33 BAG 9-10. 1991 - 5 AZR $598 / 90-$, NZA 1992, 259

34 BAG 19.6. 1991 - 5 AZR 310/90, 11.3.1992-5 AZR 237/91-, NZA 1993, 893
} 
der Bewährungszeiten bei Teilzeitbeschäftigten ${ }^{35}$. Die Frage, ob Teilzeitbeschäftigte

von Überstundenzuschlägen ausgeschlossen werden können, ist noch nicht geklärt ${ }^{36}$.

\section{Begünstigungen von aktiven Eltern, die erwerbstätig sind}

\section{Kinderbetreuung wäbrend der üblichen Erwerbsarbeitszeiten}

Der neugeschaffene Rechtsanspruch auf einen Kindergartenplatz für Kinder über drei Jahre ( $\$ 24$ Abs. I KJHG, gültig ab I. I. 1996) schweigt über Öffnungszeiten der Einrichtungen. Erfüllt ist der Rechtsanspruch z. B. auch durch Angebote für die Zeit von 9 bis I 2 Uhr, Nachmittagsangebote und Angebote für jeden zweiten Wochentag. $\$ 24$ Abs. I KJHG enthält also einen - begrüßenswerten - Bildungsanspruch für Kinder von drei bis sechs Jahren, der mit der Erwerbstätigkeit aktiver Eltern allenfalls insoweit verknüpft ist, als eine ggf. zur Ermöglichung einer Erwerbstätigkeit engagierte Betreuungsperson in der Zeit des Kindergartenbesuches Haushaltstätigkeiten erledigen kann, was erwerbstätige aktive Eltern zumindest mittelbar entlastet.

Die Pflicht zur Schaffung eines bedarfsgerechten Angebotes an Betreuungsplätzen für Kinder unter drei und über sechs Jahren sowie an Ganztagesplätzen für alle Altersgruppen ( $\$ 24$ Abs. $2 \mathrm{KJHG}$ ) ist zwar rechtsverbindlich ${ }^{37}$, schafft aber keine einklagbaren Ansprüche und wird deshalb bei knappen kommunalen Kassen nachrangig behandelt. Faktisch werden Einrichtungen auf Ganztagsbetreuung, die für aktive Eltern die Voraussetzung der Aufrechterhaltung ihrer Erwerbsarbeit sind, zugunsten der billigeren Erfüllung des »Rechtsanspruches « über kürzere Betreuungszeiten eingeschränkt.

In der Unterstützung kommunaler Bemühungen zur Bereitstellung von Kinderbetreuungseinrichtungen, die Müttern eine Erwerbstätigkeit ermöglichen, liegt ein Feld der betrieblichen Frauenförderung. Inzwischen finden sich - etwa im Einzelhandel, bei mittleren Industriebetrieben oder beim MDR - einige Kooperationsprojekte, in deren Rahmen auch auf spezifische Arbeitszeitgestaltungen - etwa Schichtdienst - eingegangen wird. Dabei werden auch Kooperationsprojekte zwischen öffentlicher Hand, Elterninitiativen und einem oder mehreren Betrieben realisiert ${ }^{38}$.

\section{Kurzfristige Freistellung zur Überbrückung von Engpässen}

Bei Gleichzeitigkeit von Erwerbstätigkeit und Kindererziehung entstehen immer wieder Friktionen, wenn das individuelle Betreuungsnetzwerk versagt. Die Krankheit der Tagesmutter, der Wasserrohrbruch in der Kita, »hitzefrei« in der Grundschule nehmen keine Rücksicht auf Erwerbspflichten der Eltern.

Die Lohnfortzahlungspflicht des Arbeitgebers nach $\$ 6$ I6 BGB umfaßt zwar auch erziehungsbedingte Verhinderungen, jedoch nicht bei längerer erziehungsbedingter Verhinderung. Zudem wird die abdingbare Vorschrift regelmäßig durch differenzierte tarifliche Beurlaubungsgründe konkretisiert, unter denen kinderbetreuungsbedingte nur eine untergeordnete Rolle spielen: Die "Niederkunft der Ehefrau « wird in der Regel mit einem Tag Sonderurlaub abgehandelt ${ }^{39}$. Auch $\$$ Is BErzGG greift

35 BAG 2. 12. 1992, AP Nr. 28 zu $\$ 23$ a BAT.

36 Vorlagebeschlusse des LAG Hamm (v. 22. IO. 1992, NZA 1993, 573) und des ArbG Bochum (20. I. 1993,

$\mathrm{AiB}$ 1993, 556) an den EuGH; siehe auch ArbG Hamburg, 21. 10. 1991, AiB r992, 164 (rk).

37 Dies betont zu Recht Fuchs, Gutachten F zum 60. Deutschen Juristentag, S. 79.

38 Nachweise Colnenc, RdA 1994, S.65.

39 Colneric, RdA 1994, S. 65 (69). 
nicht, da die Zeiträume, für die Erziehungsurlaub beansprucht wird, bindend beim ersten Antritt festzulegen sind ( $\$$ I6 Abs. I S. 1 BErzGG). Bei unerwartetem "Abspringen « einer erziehungsurlaubsberechtigten Betreuungsperson ist nur eine Verlängerung des Erziehungsurlaubs möglich ( $\$ 16 \mathrm{Abs} .32$. Hs. BErzGG) - also gerade kein "Einspringen « des bereits wieder erwerbstätigen aktiven Elternteils. Den Sonderfall der Krankheit eines Kindes regelt $\$ 45$ SGB V, der einen arbeitsrechtlichen Anspruch auf unbezahlte Freistellung für zehn Tage je Elternteil, Alleinerziehende für zwanzig Tage, gibt bei Substituierung des Lohnausfalls durch das »Kinderkrankengeld « ( $\$ 45$ Abs. I und 2 SGB V).

Diese Regelung wird als besonders fortschrittlich angesehen, weil sie einen Anreiz zur Teilung der Betreuung von Kindern zwischen beiden Erziehungsberechtigten biete $^{4^{\circ}}$. Die Praxis zeigt, daß Anreize zur Änderung der geschlechtsspezifischen Arbeitsteilung nur wenig beitragen: 1992 wurden nur zwischen 20 und $15 \%$ der "Kinderkrankentage « von Männern beansprucht ${ }^{41}$.

Beamtinnen, Beamte und ihnen gleichgestellte Landesbedienstete ${ }^{42}$ sind in einer besseren Lage: Die Beurlaubung nach $\$ 79$ a BBG und ihm entsprechenden landesrechtlichen Regelungen kann bis zum 18. Lebensjahr des Sprößlings zu jedem Zeitpunkt unabhängig von einer Festlegung acht Wochen nach der Geburt beansprucht werden.

\section{Neuerungen durch das Zweite Gleichberechtigungsgesetz}

Trotz der verbalen Schwerpunktsetzung auf der Vereinbarkeit von Familie und Beruf enthält das Zweite Gleichberechtigungsgesetz kaum Regelungen für die Privatwirtschaft, die diesen Bereich betreffen würden. Bezüge finden sich an drei Stellen im geänderten Betriebsverfassungsgesetz. Nach $\$ 45$ BetrVG a. F. sollen auf einer Betriebsversammlung auch Fragen der Frauenförderung und der Vereinbarkeit von Beruf und Familie behandelt werden können - auch bislang galten diese Themen überwiegend als zulässig ${ }^{43}$. $\$ 80$ BetrVG wurde um eine neue Nr. 2 a ergänzt, nach der auch die Förderung der Durchsetzung der tatsächlichen Gleichberechtigung von Frauen und Männern zu den allgemeinen Aufgaben des Betriebsrates gehört. Dies schließt Maßnahmen zur »Vereinbarkeit« ein, denn sie sind Voraussetzung tatsächlicher Gleichstellung ${ }^{44}$. Nach $\$ 93$ BetrVG n. F. soll der Betriebsrat künftig anregen können, Arbeitsplätze innerbetrieblich auch zur Besetzung durch Teilzeitbeschäftigte auszuschreiben. Nach $\$ 96$ Abs. 2 BetrVG n.F. müssen Arbeitgeber und Betriebsrat bei der Förderung der Berufsbildung der Arbeitnehmer u. a. die Belange von Arbeitnehmern mit Familienpflichten berücksichtigen.

Diese Konzentration der Reform auf Betriebsratsrechte erweckt den Eindruck, der Bundesgesetzgeber sehe die alleinige Ursache der fortwährenden Benachteiligungen von aktiven Eltern in der Privatwirtschaft in der unzureichenden Aktivität der Betriebsräte - trotz vorherrschender Orientierung vieler Betriebsräte auf überwiegend

40 Vgl. Colneric, RdA 1994, S.65 (73).

${ }_{11}$ Pınl, Das faule Geschlecht, I994, S. I 76 nach einer schriftlichen Auskunft des Bundesministeriums für Gesundheit.

42 In Berlin, Bremen, Hessen, Niedersachsen, Mecklenburg.

43 Die Fragen der Gleichstellung von Frauen und Männern werden als zulässiges Thema ausdrücklich erwahnt von Wlotzke, BetrVG, 2. Aufl 1992, Anm 2 zu $\$ 45$; Dietz, Richardi, BetrVG, 6. Aufl 1982 , Rz 10, Futtung, Auffahrt, Kaiser, Heither, BetrVG, 17. Aufl I992, Rz 10; Fragen der Kinderbetreuung erwähnen Daubler, Ktttner, Klebe, Schneuder, BetrVG, 4 . Aufl 1994, Rz 3 zu $\$ 45$ sowie Löwisch, TKBetrVG, Rz 2 zu $\$ 4$ s. Weder Frauenförderung noch Kinderbetreuung erwähnen Hess, Schlochauer u. a., BetrVG, 4. Aufl 1992.

44 Birk hält demgegenüber eine gesonderte Verpflichtung des Betriebsrates, sich für familienfreundlichere Arbeitsbedingungen einzusetzen, für erforderlich, Gutachten E zum 60. DJT, S. 86, 94. 
männliche Interessen eine sicherlich unzutreffende Analyse. Die Regelungen des Zweiten Gleichberechtigungsgesetzes zur Vereinbarkeit für den öffentlichen Dienst beschränken sich auf die graduelle Verstärkung der Ansprüche auf Beurlaubung und Reduzierung der Arbeitszeit im Beamtenrecht sowie einige Empfehlungen zur flexiblen Arbeitszeit für den Bereich der Bundesverwaltung. Die Benachteiligungsverbote für Teilzeitbeschäftigte in der Bundesverwaltung bleiben hinter denen des EG-Rechts zurück. Hier zeigt sich, daß das Zweite Gleichberechtigungsgesetz auch familienpolitisch alles andere als ein Bravourstück ist ${ }^{45}$. In seiner Unzulänglichkeit ist es Symptom der politischen Ratlosigkeit gegenüber den Friktionen zwischen Mutterschaft und Erwerbswelt. Schließlich sind auch die Regelungen der Landesgleichstellungsgesetze - überwiegend aus sozialdemokratisch regierten Ländern nicht wesentlich phantasievoller.

\section{B. Erfabrungen mit der "Vereinbarkeit"}

\section{Die gnädige Unklarbeit des Begriffs}

Was eigentlich mit dem Schlagwort »Vereinbarkeit von Familie und Beruf « genau gemeint ist, wird denn auch in der politischen Diskussion kaum spezifiziert ${ }^{46}$. Aufgrund dieser gnädigen Unklarheit können mit dem Begriff höchst unterschiedliche familien- und gleichstellungspolitische Vorstellungen assoziiert werden.

Wegen unzureichender außerhäuslicher Betreuungseinrichtungen für Kleinkinder ist eine Vollerwerbstätigkeit bei aktiver Elternschaft heute faktisch kaum möglich. Um Erwerbstätigkeit beider Eltern ohne Einstellung einer Kinderfrau zu ermöglichen, müßten solche Einrichtungen von sieben bis ig Uhr geöffnet sein und außerdem die Möglichkeit der Rund-um-die-Uhr-Betreuung für Dienstreisen, Fortbildungen und Schichtarbeit bieten - und das auch in den Schulferien. Die Bundesrepublik ist insoweit bekanntlich Entwicklungsland ${ }^{47}$ : Der Versorgungsgrad für Kinder bis zu drei Jahren lag Ende I99I in den alten Bundesländern bei 2,7\%, für Kinder von 3 bis 6 Jahren bei $69 \%$, in den neuen Bundesländern bei knapp zwei Dritteln (unter Dreijährige) bzw. $95 \%$ (Drei- bis Sechsjährige) ${ }^{48}$. In der DDR herrschten noch 1988 rein quantitativ utopische Zustände - der Versorgungsgrad mit Kindereinrichtungen für die Altersgruppe $\circ$ bis 3 Jahre betrug $79 \%$, für Drei- bis Sechsjährige über $100 \%{ }^{49}$. In Belgien und Frankreich gibt es zumindest für 20-25\% der Kinder unter drei Jahren Betreuungsplätze und in Dänemark für $44 \%^{50}$. Auch gegenüber den häufig pauschal als »rückständig « betrachteten südlichen Mitgliedsstaaten der EU schneidet die Bundesrepublik nicht günstig ab: $97 \%$ aller griechischen Kinder im Alter von drei bis sechs Jahren besuchen einen Ganztagskindergarten" ".

Die Überzeugung, eine professionelle Betreuung in Gruppen sei auch für Kleinkinder ein Gewinn, ist außerdem in Deutschland nicht weit verbreitet. Für die Auffassung, es sei »nicht nur biologisch, sondern auch sozial (...) geboten, das sich ein Elternteil in der für die gesamte spätere Entwicklung entscheidenden ersten Lebensphase des Kindes ohne Belastung aus einem Arbeits- oder Dienstverhältnis der

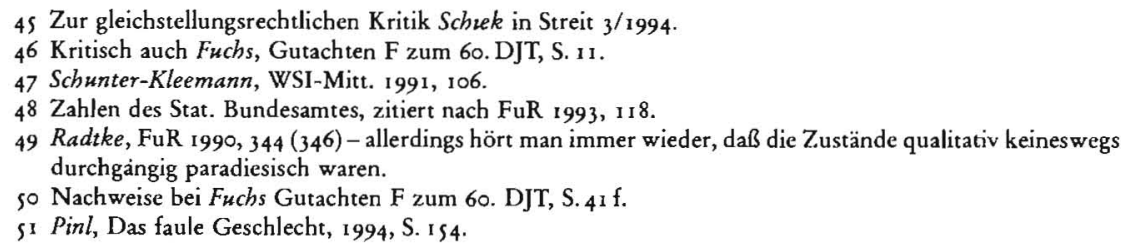


Betreuung und Erziehung des Kindes widmet ${ }^{{ }^{52}}$, werden nicht einmal Belege zitiert's, Nach arbeitsrichterlicher Auffassung gehört es gar »zu den gesicherten Erkenntnissen der Entwicklungspsychologie und soziologischen Sozialisationsforschung, daß der mütterliche ${ }^{54}$ Einfluß im frühen Stadium der Entwicklung von entscheidender Bedeutung ist «s. Mit dem Bundeserziehungsgeldgesetz ${ }^{56}$ sind solche Auffassungen zum Gesetzesziel geworden ${ }^{57}$.

Das Ideal der Hausfrauenmutter wird in der deutschen Bevölkerung besonders stark vertreten. In einer repräsentativen Untersuchung ${ }^{8}$ gaben $36 \%$ der deutschen Männer und $26 \%$ der deutschen Frauen an, auch eine Mutter von Schulkindern solle gar nicht erwerbstätig sein. In den USA fanden dies nur 6,7\% der Männer und $\mathbf{4}_{, 2} \%$ der Frauen; in Großbritannien 6,3\% der Männer und 3,4\% der Frauen. Die Erwerbstätigkeit der Mütter von Vorschulkindern lehnen 70,1\% der deutschen Männer und $61,8 \%$ der deutschen Frauen gegenüber $50,3 \%$ der US-amerikanischen Männer und $39,3 \%$ der US-amerikanischen Frauen sowie $65,7 \%$ der britischen Männer und $54,4 \%$ der britischen Frauen ab.

Sowohl mangels öffentlicher Betreuungsmöglichkeiten als auch aufgrund der nationalen Moralvorstellungen werden die meisten deutschen Kinder familiär erzogen. Nach Ablauf des gesetzlichen Mutterschaftsurlaubs bleiben $95 \%$ der Mütter und I,6\% der Väter zu Hause, 3\% der Kinder werden außerhäuslich oder durch Angestellte im Haus betreut. Auch nach Beendigung des Erziehungsurlaubs nehmen nur $50 \%$ der Mütter die Erwerbstätigkeit wieder auf, davon ca. I/ 3 in Teilzeitarbeit. Diese Zahlen haben sich seit der Einführung des Mutterschaftsurlaubs 1979 nicht wesentlich geändert ${ }^{59}$. Allerdings streben auch die übrigen Frauen die Wiederaufnahme der Erwerbstätigkeit zu einem späteren Zeitpunkt an.

Vorherrschend ist damit immer noch das Phasenmodell, allerdings nicht mehr mit drei $^{60}$, sondern mit vier Phasen: Nach einer Vollzeittätigkeit zur Ausbildung und zum Erwerb erster Berufserfahrung (Phase 1 - Erstberufstätigkeit) gebiert die Frau ein oder mehrere Kinder mit der Folge von Mutterschutz und Erziehungsurlaub, ggf. verlängert durch weitere Freistellung (Phase 2 - Vollzeithausfrau und -mutter), nimmt anschließend - bei Versorgung des Kindes in einer Kleinsteinrichtung, durch eine Tagesmutter oder Familienangehörige - eine Teilzeittätigkeit auf (Phase 3 Wiedereinstieg als Teilzeithausfrau und -mutter) und kehrt schließlich, wenn der

52 Buglass/Helmann, AuR 1992, 353 (356).

53 Belege für die gegenteilige Auffassung, daß Wohlergehen eines Kindes auch bei Erwerbstätigkeit der Mutter gewahrleistet werden kann, bei Körner-Damman, NJW 1994, 2056 (2062).

54 Hervorhebung von mir.

ss ArbG Saarbrücken, 20. Ix. 1987, EzBAT Nr 4 zu $\$$ so BAT Unbezahlter Sonderurlaub.

s6 Vom 6. Dezember 1985, BGBl. I S.2154, in der Fassung der Bekanntmachung vom 21. Januar 1992 (BGBI. I S. 68, zuletzt geandert durch Gesetz vom II. Januar 1993, BGBI. I S. 50).

s7 Vgl. Regierungsbegrundung BR-Drs. 350/85, S. 13, wo zum Ziel des Gesetzes erklärt wird, daß »die Mutter weiterhin vorrangig zu Hause bleibt«, nicht ohne zu betonen, daß »durch die Möglichkeit der Erziehungsleistung durch den Vater die Wahlfreiheit der Eltern, wer das Kind betreuen soll, vom Gesetz. anerkannt $\%$ wird.

s8 Alwin, Braun, Scott, European Sociological Review, 1992, I 3.

59 Die Zahlen zum Erziehungsurlaub stutzen sich auf den ersten und einzigen Bericht der Bundesregierung zu den Erfahrungen mit dem Bundeserziehungsgeldgesetz für den Zeitraum 1987 bis 1988 (BT-Drs. I I/8517, zusammenfassende Darstellung Lemke, FuR 1991, 83), die ihm zugrundeliegende Studie des Instituts für Entwicklungsplanung und Strukturforschung (veröffentlicht in den Teilberichten »Betriebliche Wirkungen des Erziehungsurlaubs" (Hannover 199r), Erziehungsgeld und Erziehungsurlaub als Bestandteil der Sozıalen Marktwirtschaft (Tagungsdokumentation Hannover 1991), Situation Erziehungsurlaub, Erziehungsgeld - Zur Lage der Alleinerziehenden (Hannover 1991)) und die Vorläuferstudie zu den Auswirkungen des Mutterschaftsurlaubs (Weg u.a., Erwerbstatigkeit und Mutterschaft, Schriftenreihe des BMA Bd. 136, 1986).

6o Der Begriff "Drei-Phasen-Modell « wurde von Myrdal, Klein als Beschreibung der Lebensrealität von Müttern in den siebziger Jahren geprägt; dies., Die Doppelrolle der Frau in Familie und Beruf, 3. Aufl. 1971. 
Arbeitsmarkt es zuläßt, wieder vollzeitig ins Erwerbsleben zurück (Phase 4 - vollständiger Wiedereinstieg) ${ }^{6 t}$.

Vereinbarkeit im Wortsinn - d.h. Gleichzeitigkeit von Erwerbstätigkeit mit branchenüblicher Arbeitszeit und Elternschaft - kann nur eine Minderheit von Müttern realisieren, während sie für Väter nach wie vor der Normalfall ist. Sogar alleinerziehende Frauen nehmen vielfach eine Unterbrechung der Erwerbstätigkeit in Kauf und sind in der Folge häufig auf Sozialhilfe angewiesen ${ }^{62}$. Gleichzeitigkeit von aktiver Elternschaft und Erwerbstätigkeit ist immer labil: Selbst da, wo eine preisgünstige öffentliche Einrichtung mit sechs bis achtstündiger Öffnungszeit involviert ist, sind weitere Personen zur Betreuung vor Beginn und nach Ende der Öffnungszeit sowie für den Fall von Krankheit und abendlichen Freizeitaktivitäten erforderlich. Diese kann die Alleinerziehende nicht immer (angemessen) vergüten, so daß sie auf Verwandte oder engagierte Freunde angewiesen ist ${ }^{63}$.

Man sollte meinen, daß bei aller Orientierung der Vereinbarkeitsdiskussion an der vierphasigen Versorgerehe das Zweite Gleichberechtigungsgesetz wenigstens zur Absicherung dieses Modells beiträgt.

Dies ist aber nicht der Fall. Der Erziehungsurlaub sichert aktiven Eltern den Übergang von Phase 2 (Vollzeithausfrau/mann) in Phase 4 (volle Erwerbstätigkeit). Die weitergehenden beamtenrechtlichen Beurlaubungs- und Ermäßigungsmöglichkeiten sichern darüber hinaus den Übergang von Phase $2 \mathrm{zu}$ Phase 3 (Teilzeithausfrau/mann) und von Phase 3 zu Phase 4 . Die Nachteile der Phasen zwei und drei für die berufliche Entwicklung werden nur für Beamtinnen und Beamte relativ komfortabel kompensiert.

Für Beschäftigte in der Privatwirtschaft ist die Durchführung des Modells weiterhin ungesichert. Sie müssen den Übergang von Phase 2 in Phase 3 und von Phase 3 in Phase 4 individuell aushandeln. Tarifverträge und Betriebsvereinbarungen enthalten bestenfalls Vorrangregelungen. Gesichert ist damit allenfalls der Ausstieg aus der Erwerbstätigkeit qua Erzichungsurlaub. Zu dessen Beginn können sich aktive Eltern noch in der Illusion wiegen, nach seinem Ende wieder erwerbstätig zu werden. Mit einer Wahrscheinlichkeit von $50 \%$ gelingt dies jedenfalls den Müttern nicht - Resultat einer Kombination von Frauen- und Elterndiskriminierung. Angesichts des großen Interesses junger Mütter (das Interesse der Väter hält sich noch in Grenzen) an Teilzeitarbeit darf man annehmen, daß ein wesentlicher Grund dafür die mangelnde Absicherung des Überganges in Phase 3 ist.

\section{II. Ökonomische Sicherung aktiver Eltern}

Die ökonomische Sicherung aktiver Eltern wird üblicherweise nicht diskutiert, im Zentrum der familienpolitischen Diskussion steht vielmehr die Sicherung der Familie als Ganzer. Frauen wird mit dieser Debatte zugemutet, mit der Entscheidung für ein Kind die wirtschaftliche Unabhängigkeit aufzugeben ${ }^{64}$. Den wenigen aktiven Ausnahmevätern steht diese Option meist nicht offen, da die wenigsten Frauen genug verdienen, um eine Familie zu ernähren. Der Verweis auf den Verdienst des

6I Dieses Phasenmodell ist nicht nur Realitätsbeschreibung, sondern auch gemeinsames Idealbild christdemokratischer (vgl. Begrundung des Gleichberechtigungsgesetzes) und (real)gruiner Familienpolitik (vgl "Strategien zur beruflichen Integration der Frauen in Niedersachsen «, 1991, im Auftrag von Waltraud Schoppe); auch das Elternarbeitszeitgesetz der SPD-Bundestagsfraktion (B'T-Drs. I $1 / 8023$ ) hat es auf seine Fahnen geschrieben.

62 Vgl. Nave, Kriger, IFG Materialien zur Frauenforschung, Bd. 15 , 1992, S. 45.

63 Zur Bedeutung von sozialen Netzen fur unabhängige Mütter vgl Nıepel, IFG Materialien zur Frauenforschung, Bd. is, 1992, S. 60.

64 Kritisch Fucbs, Gutachten F zum 60. DJT, S. 68 
Mannes ist um so fataler, als die wirtschaftliche Sicherung durch eine Ehe kein verläßliches Fundament (mehr) ist. Bei der Scheidung sind die Unterhaltsansprüche meist für eine wirtschaftliche Sicherung von Mutter und Kind nicht ausreichend; Sozialhilfeabhängigkeit ist eine häufige Folge ${ }^{65}$. Obwohl die meisten Scheidungen von Frauen eingereicht werden, ist es nicht ausgeschlossen, daß Frauen aufgrund dieser ökonomischen Situation auch in nicht mehr gewünschten Beziehungen ausharren. Was das frühkindliche Erleben dieser persönlichen Abhängigkeit der Mutter für die Orientierung von Mädchen bedeutet, ist gar nicht auszudenken. Vor allem aber erinnert die Existenzsicherung Erwachsener durch eine persönliche Beziehung auf der Grundlage ökonomischer Abhängigkeit an feudale Strukturen und kann kein Leitbild einer entwickelten Gesellschaft sein. Die ökonomische Absicherung der Mutter - selbstverständlich mit ihren Kindern - ist deshalb auch jenseits der Erwerbstätigkeit ein eigenständiger Wert.

Wenn Vereinbarkeitsregeln keine Einkommensersatzleistungen für Eltern vorsehen, die sie in Anspruch nehmen wollen, zeigt dies ihre gesellschaftspolitischen Implikationen: Angestrebt ist nicht ökonomische Eigenständigkeit von aktiven Eltern, sondern die Sicherung der Erfüllung der Hausfrauenmutterrolle wenigstens für eine kurze Phase, da dies freiwillig nicht immer gewährleistet scheint. Das Vier-PhasenModell wird nur auf der Grundlage einer Versorgerehe (oder modifizierten Versorgerehe ${ }^{66}$ ) ermöglicht. Implizit gehen seine Rahmenregelungen davon aus, daß Frauen weiterhin bereit sind, sich mit der Mutterschaft in die ökonomische Abhängigkeit vom »Partner « zu fügen. Die ökonomische Unabhängigkeit von Müttern kann nur durch die Gleichzeitigkeit von Erwerbstätigkeit und Mutterschaft sichergestellt werden. Selbst wenn das Erziehungsgeld als Einkommensersatzleistung ausgestaltet wäre, wäre es doch illusorisch zu erwarten, daß irgendeine öffentliche oder Versicherungskasse eine solche Leistung länger als ein bis zwei Jahre pro Kind gewähren kann. Im Anschluß müßte die Möglichkeit der Erwerbstätigkeit gegeben sein. Vereinbarkeit im Sinne von Gleichzeitigkeit ist auch deshalb unverzichtbar.

\section{II. "Phasenideologie und Arbeitsmarktposition von Frauen}

\section{Gescblechtsspezifische Wirkung des Vereinbarkeitsrechts am Arbeitsmarkt}

Die Vereinbarkeit von aktiver Elternschaft und Erwerbstätigkeit kann nicht losgelöst von der Arbeitsmarktsituation der Frauen betrachtet werden. Da die Männer unter den aktiven Eltern so unterrepräsentiert sind, haben Vereinbarkeitsprobleme auf die Arbeitsmarktlage der Männer als Gruppe keine negativen Auswirkungen. Am Arbeitsmarkt ist die Vereinbarkeitsdiskussion eine reine Frauenfrage.

Solange Frauen in ihrer Mehrheit die weniger qualifizierten, geringer entlohnten, schlechter geschützten Arbeitsplätze innehaben, bleibt selbst die Möglichkeit der Entscheidung zwischen Familie und Erwerbstätigkeit für viele ein Wunschtraum: Eine eigenständige Existenz auf gesellschaftlichem Durchschnittsniveau ist für sie aus eigener Erwerbstätigkeit ohnehin nicht erreichbar. Die Entscheidung für die ökonomische Abhängigkeit in einer (modifizierten) Versorgerehe erlaubt dann wenigstens die Realisierung eines Kinderwunsches. Auch setzt die Gleichzeitigkeit von Mutterschaft und Erwerbstätigkeit ein ausreichendes Angebot bezahlter Arbeitsplätze voraus. Damit sind hier nicht nur Teilzeitarbeitsplätze gemeint ${ }^{67}$. Generell ist das Arbeitsplatzangebot für Frauen geringer als das für Männer, auch wenn in eini-

65 So auch Korner-Dammann, NJW 194, S. $2056(2058)$.

66 Begriff Getssler, Pfan, WSI-Mitt. 1989, S. I 88.

67 So Fuchs, Gutachten F zum 60. DJT, S. 40. 
gen Regionen in jüngster Zeit die Männerarbeitslosigkeit schneller gestiegen ist als

die Frauenarbeitslosigkeit ${ }^{68}$.

\section{Situation von Müttern, die die Regelungen der "Vereinbarkeit in Anspruch nebmen}

Die Verwirklichung des Vereinbarkeitskonzepts durch Erziehungsurlaub, längere "Familienpause", anschließende Teilzeitarbeit bis zum Ende der Schulpflicht ist mit erheblichen beruflichen Nachteilen verbunden. Während der Beurlaubung geht die Verbindung zum Beruf zumindest teilweise verloren, Dequalifizierung tritt ein. Sie wird auch bei einfachen Tätigkeiten von vielen Unternehmen schon bei einer Unterbrechung von ein bis zwei Jahren gesehen ${ }^{69}$. Fuchs beziffert die rein ökonomischen Verluste einer Frau (oder eines Mannes) durch eine zehnjährige "Familienpause " auf 430000 bis 800000 Mark, durch einen dreijährigen Erziehungsurlaub auf 163100 bis $297000 \mathrm{Mark}^{70}$.

Den Vorteilen, die Teilzeitarbeit für Arbeitgeber haben mag - höhere qualitative und quantitative Arbeitsleistung, geringere Fehlzeitquote, günstige Bewältigung wiederkehrender Arbeitsspitzen, Gewährleistung notwendiger Kapazitätsauslastung bei verkürzter Regelarbeitszeit ${ }^{71}$-, stehen reziproke Nachteile auf Seiten der Teilzeitbeschäftigten gegenüber: Arbeitsverdichtung, die besonders in Kombination mit Tätigkeit im Haushalt zu verstärkter Belastung führt, und Einkommensverlust, ungünstiges Verhältnis von Wegezeiten zur Arbeitszeit und Verlust betrieblicher Kommunikationsmöglichkeiten. Der sozialrechtliche Nachteilsausgleich läßt ebenfalls zu wünschen übrig ${ }^{72}$. Die Folge sind weitgehender Ausschluß der »Familienfrauen " von Leistungen der Arbeitsförderung ${ }^{73}$ und erhebliche Defizite bei der Alterssicherung.

Mit dem Einlassen auf eine zeitweise Hausfrauenehe übernimmt die »Vereinbarkeitlerin « nicht nur die Erziehungsarbeit, sondern in der Regel alle Haushaltspflichten. Mit dem Übergang in Teilzeitarbeit wird ihr ermöglicht, die vollständige Verantwortung für die Haushaltsführung - in besser gestellten Haushalten durch eine Haushaltshilfe unterstützt - weiterhin ohne Unterstützung des »Partners « zu tragen. Daß dieser nach Beendigung der Familienphase seinen Anteil übernimmt, wird aufgrund der langen Gewöhnung beider an das Arrangement zunehmend unwahrscheinlich. Die Mutterschaft gilt deshalb als das Unterpfand, mit dem eine geschlechtsspezifische Arbeitsteilung auch bei im Grunde emanzipierten Frauen durchsetzbar ist. Die Belastungen der Frauen werden verstärkt und die Männer wirksam von Familienarbeit entlastet ${ }^{74}$. Drastisch wurde diese Gefahr bei der Anhörung zum Gleichberechtigungsgesetz geschildert:

"Bei der Familienförderung, wenn ich sie nur in Richtung Frauen adressiere, gibt es die Gefahr daß ich (.) es (nur) den Frauen ermögliche, ihren tradierten Pflichten (.) gerecht zu werden. Dann zappeln sie sich überall ab. So bei den Teilzeitbeschäftigten

68 Naher Bundesanstalt fur Arbeit, Berufliche Bildung und Beschaftigung von Frauen, 1994.

69 Pfarr, ZRP I 994, S. 309 (311).

70 Gutachten F zum 60. DJT, S. 46 (und eigene Berechnungen).

71 Lipke, AuA 1994, S. 8 m.w. N.

72 Vgl. Fuchs, Gutachten F zum 60. DJT, a. a. O.

73 Hierzu Däubler, Gleichberechtigung und Arbeitsforderungsgesetz, 2. Aufl. 1992.

$74 \mathrm{Vgl}$. Marsh, Catherine, Hours of Work of Women and Men in Britain, 1991, S. 80: „It might be useful to consider regulation of the hours of work of men as the single most effective means of promoting equality at the workplace. Rearranging hours of work to make them more convenient for women is likely merely to increase their total work burden once domestic labour has been taken into account. It might be better to tackle the work schedules of men which make it impossible for them to take a share in running the home and caring for children.* 
in extremer Weise durch die Leistungsverdichtung. Diese ackern nämlich in den paar Stunden, die sie im Betrieb sind, wie die Wilden mit relativ reduzierten Kommunikationsmöglichkeiten, und zum anderen rasen sie dann nach Hause und bringen den Haushalt auf Vordermann, damit die Pampuschen vorgewärmt sind, wenn nachher der Alte nach Hause kommt ${ }^{75}$.

Pilotstudien lassen den vorsichtigen Schluß zu, daß Teilzeitfrauen mit "Familienpflichten « zu den am stärksten belasteten Arbeitnehmergruppen gehören, betrachtet man die rein zeitliche Arbeitsbelastung. Daraus ergeben sich bedenkliche Gesundheitsbelastungen ${ }^{76}$.

\section{Auswirkungen des Vierphasenmodells auf die Arbeitsmarktposition von Frauen}

Aber nicht nur die Mütter, die dem gesetzlich empfohlenen Vereinbarkeitsmodell folgen, müssen Nachteile hinnehmen. Solange "Vereinbarkeit« sich auf einen unzulänglichen Nachteilsausgleich bei Unterbrechungen der Erwerbsarbeit zur höchstpersönlichen familiären Kinderbetreuung beschränkt, bleibt die Möglichkeit des Wechsels zwischen Erwerbs- und Familientätigkeit Personen vorbehalten, die ihre Existenz anderweit sichern können. Die Möglichkeit hierzu haben durch Unterhaltsansprüche gesicherte Frauen. Wegen der ungleichen Arbeitsmarktchancen der Geschlechter sind Männer höchst selten insoweit »versorgt«. Objektiv können sich die meisten Familien eine andere Aufteilung aufgrund der weit überproportionalen Verdienstchancen von Männern und der symbolischen finanziellen Entlastung bei Erziehungsurlaub gar nicht leisten. Schon deshalb werden Vereinbarkeitsregelungen auch in Zukunft im wesentlichen von Frauen beansprucht werden.

Die Vorherrschaft der durchbrochenen Berufsbiographie von Müttern wirkt sich nachteilig auf die Arbeitsmarktposition aller Frauen aus. Zum einen verfestigen sich mit der geschlechtsspezifischen Arbeitsteilung in Partnerschaft und Familie Arbeitsmarktvorteile von Männern. Sie sind es, die auf eine stabile Entlastung von Versorgungstätigkeit vertrauen können, wodurch ihnen die flexible Reaktion auf Arbeitgeberbedürfnisse ermöglicht wird. Sie können für kurzfristige Überstunden oder notwendige Fortbildungen zur Verfügung stehen und ihr stabilisierendes Umfeld auch bei Wechseln des Arbeitsortes bei sich behalten. Sie können sich auf den Beruf konzentrieren. Frauen dagegen bleiben auch bei Vollzeitbeschäftigung mindestens mit ihrer eigenen Versorgung, meistens noch mit der Versorgung eines anderen Erwachsenen und ggf. gemeinsamer Kinder belastet. Diese Belastung schränkt ihr berufliches Engagement ein. Am stärksten beeinträchtigt sind teilzeitbeschäftigte Familienfrauen, die offensichtlich versuchen, den Anforderungen beider Arbeitsbereiche vollständig zu genügen. Überspitzt ausgedrückt: Jede Frau, die einen Mann hauswirtschaftlich, emotional und sozial versorgt, beeinträchtigt ihre eigenen und ihrer Geschlechtsgenossinnen Arbeitsmarktchancen, weil sie einen weiteren für Frauen (und Männer, die einseitige kostenlose Dienstleistungen gerade in einer persönlichen Beziehung ablehnen) unschlagbaren Konkurrenten schafft. Zum anderen beeinträchtigt die Unvereinbarkeit von üblicher Erwerbstätigkeit und Mutterschaft die Arbeitsmarktchancen aller Frauen. Bei Frauen besteht die biologische Wahrscheinlichkeit der Mutterschaft. Mutterschaft führt zum Mutterschutz mit finanziellen Belastungen für den Arbeitgeber und - aller statistischen Erfahrung nach - zum

$75 v$ Friesen, Anhorung des Bundestagsausschusses für Frauen und Jugend zum Entwurf eines Zweiten Gleichberechtigungsgesetzes (BT-Drs. 12/5468) und dem Entwurf eines Gleichstellungsgesetzes der Fraktion der SPD (BT-Drs. I 2/5717) am I1 I I. 1993, Stenographisches Protokoll, S. 72.

76 Elsner, AiB 1989, S. x 56 ; Funder, in: Klein (Hrsg.) Nicht immer, aber immer öfter - Flexible Beschaftigung und ungeschitzte Arbeitsverhaltnisse, 1993, S. 26. 
mindestens zeitweisen Ausscheiden der Mutter aus dem Arbeitsverhältnis. Indem sie keine Frauen beschäftigen, können Arbeitgeber lästige Beurlaubungsansprüche vermeiden. Im Zeitraum von 1983 bis 1988 hatten immerhin $18,8 \%$ der Betriebe keine Ausfälle durch Mutterschutz und Erziehungsurlaub - in diesen Betrieben war der Frauenanteil signifikant unterdurchschnittlich ${ }^{77}$. Obwohl der Anteil der »Erziehungsurlauberinnen « je Ioo beschäftigte Frauen mit ca. $3 \%$ seit 1980 relativ konstant niedrig geblieben ist, wird schon die Möglichkeit von Unternehmensseite als Beeinträchtigung der Souveränität empfunden ${ }^{78}$. Sicherlich spielen dabei auch Vorstellungen über die Präokkupation jeder Mutter mit Haushalt und Kindern eine Rolle. Auch wenn Männer wegen Karrierewünschen den Arbeitgeber häufiger wechseln sollten als Frauen, so besteht bei ihnen doch die Chance, sie durch bessere Angebote in der Firma zu halten - während Frauen objektiv statistisch gesehen und unabhängig vom individuellen Kinderwunsch unstete Arbeitskräfte sind, die ggf. ganz aus dem Erwerbsleben scheiden. Es kann ökonomisch sinnvoll erscheinen, sie auf Positionen einzusetzen, die keine Einarbeitungszeit voraussetzen - am Ende der betrieblichen Hierarchie. Die Mutation der Frau zur stetigen Arbeitskraft setzt Vereinbarkeit von Mutterschaft und Erwerbstätigkeit voraus, denn die meisten Frauen werden immer noch Mutter. Die realistische Erkenntnis, daß das größte Arbeitsmarkthindernis für Frauen ihre Gebärfähigkeit ist, hat allerdings in den neuen Bundesländern schon zu pressewirksamen Sterilisierungskampagnen von Arbeitnehmerinnen geführt 79 .

\section{Reformperspektiven}

\section{Verbesserte Absicherung des Vierphasenmodells?}

Zahlreiche Reformvorschläge im Zusammenhang mit der Vereinbarkeit konzentrieren sich auf eine bessere Absicherung des Vierphasenmodells. So schlugen Pfau/ Geissler $^{8 \circ}$ vor, den Erziehungsurlaub durch ein Zeitkonto für Eltern, gestaffelt in Vollfreistellung und Teilzeitarbeit, mit einer Sozialleistung zur Existenzsicherung zu ersetzen. Männer sollten an diesen Regelungen partizipieren können, aber nicht müssen. Faktisch würde dies sicher darauf hinauslaufen, daß Frauen das »Müttergehalt « als Verbesserung ihrer Situation nutzen. Auch Matthies $u \cdot a \cdot{ }^{81}$ plädieren für eine Einkommensersatzleistung zur Existenzsicherung im »Elternurlaub«. Ein Zeitkonto im Erziehungsurlaub - allerdings ohne jede materielle Absicherung oder gar Teilzeitoption - schlägt auch Birk ${ }^{82}$ vor. Für den öffentlichen Dienst will er auch Arbeitnehmerinnen und Arbeitnehmern einen Anspruch auf Teilzeit geben.

All diese Vorschläge würden die Situation verbessern, denn sie würden das Vierphasenmodell stärker absichern und damit den bei der gegenwärtigen Diskriminierung am Arbeitsmarkt verständlichen Wünschen von Frauen entgegenkommen. Dem Mechanismus, daß bei schlechter Arbeitsmarktlage der Einstieg gerade der Mütter in

77 Spell (Bearb.), Betriebliche Wirkungen des Erziehungsurlaubs, Erfahrungen mit der Anwendung des Bundeserziehungsgeldgesetzes, Material Nr. I I I des Institutes für Entwicklungsplanung und Strukturforschung, 1991, S. 15. Am höchsten war dieser Anteil im Handwerk (19,4\%), am niedrigsten bei den nichtkommerziellen Dienstleistungen $(9,1 \%)$.

78 Ebenda, S. 17; Weg u. a., Erwerbstatigkeit und Mutterschaft (Kurzfassung), 1987, S. 5, nannte fur den Zeitraum von 1980 bis 1984 noch einen Anteil von $4 \%$.

79 Von ausdrucklichen Verlangen nach Sterilisation berichtet Coester, Anm. BAG AP Nr. 8 zu $\$ 6$ I I a BGB, $\mathrm{Bl}>\mathrm{R}$.

80 Im Rahmen eines Gutachtens fur die Bundestagsfraktion der Grünen, Auszuge veröffentlicht in WSIMitteilungen 1989, 188; s.a. DIE GRUNEN, Argumente: Geschützte Teilzeit fur Eltern, und BeckOberdorf u. a. und die Fraktion der Grinen, Entwurf eines Gesetzes zur Freistellung und Reduzierung der Erwerbsarbeitszeit für Erziehende - Zeit für Kinder, BT-Drs. I1 $1 / 8423$

81 Arbeit 2000,1994, S. $299 \mathrm{f}$

82 Gutachten E zum 60. DJT, S. 69. 
Phase 2 zum endgültigen Ausstieg aus der Erwerbsarbeit wird, könnten sie zum Teil entgegenwirken. Mit Ausnahme der Vorschläge von Geissler/Pfau sowie Matthies u. a. tragen sie nichts zur ökonomischen Sicherung aktiver Eltern bei. Ganz ohne materielle Absicherung wird aber der Anteil der Männer an den aktiven Eltern nicht einmal um die paar Gutwilligen steigen, die bereit sind, auf ihre Privilegien zu verzichten und dazu noch den Spott zu ertragen.

Keines der Modelle ändert jedoch effektiv die Zuordnung des »Kinderrisikos « zur weiblichen Arbeitskraft. Da die meisten Frauen ihre Kinder in Partnerschaften bekommen, erhalten alle diese Modelle auch die Hausfrauenehe: abgesichert durch die Freistellung, bleibt frau nach der Geburt zu Hause. Eine Umkehr der einmal eingespielten häuslichen Arbeitsteilung gelingt meistens nicht. Die Arbeitsmarktnachteile, die für alle Frauen aus dem Erziehungsurlaubssystem resultieren, gehen also auch mit diesem Modell einher. Die ökonomische Sicherung während der Phasen 2 und 3 kann die ökonomischen Risiken, denen die Mütter dann aufgrund der Arbeitsmarktlage ausgesetzt sind, voraussichtlich nicht ausgleichen. Hierdurch wird wieder der Trend zur Versorgung durch wirtschaftlich Stärkere gestärkt: Die modifizierte Versorgerehe bleibt die ökonomisch sinnvollste Wahl.

Gerade die Teilzeitoption stabilisiert aber wieder die Arbeitsteilung in herkömmlichen »Partnerschaften « zuungunsten der Frauen und verweist sie wegen der mangelhaften ökonomischen Absicherung gleichzeitig auf die herkömmliche "Partnerschaft«. In der Arbeitsteilung in diesen Partnerschaften muß eine wesentliche Ursache für das Scheitern der Reintegration von Müttern in die Erwerbstätigkeit gesehen werden. Heute wird die reine Hausfrauenehe ohne Kinder immer weniger akzeptiert. Sogar die Deutschen fanden in der o.g. Umfrage nur zu $8 \%$ (Frauen $6,4 \%$ ), daß in einer Ehe ohne Kinder die Frau zu Hause bleiben soll. Erst mit der Geburt von Kindern hat der Ehemann die Möglichkeit, alle Dienstleistungen einer Hausfrau zu beanspruchen.

Die Rückkehr der Mutter zur Normalarbeitszeit bedroht dieses Arrangement. Den Konflikten aufgrund der mangelnden Akzeptanz der Zurückhaltung von Haushaltsdienstleistungen durch ihren "Partner " können Frauen entgehen, indem sie als Teilzeitbeschäftigte bei hälftigem Verdienst sowohl im Beruf als auch im Haushalt in der halben Zeit die volle Leistung erbringen. Da eine Vollerwerbstätigkeit irreversible Einschränkungen der Haushaltsbequemlichkeit mit sich bringt - die Frau kann kein Mittagessen kochen und nicht vor Heimkehr des Mannes die Aufräum- und Putzarbeiten erledigen - werden nur wenige Frauen zu ihr zurückkehren. Ob die durchgehende und chancengleiche Erwerbstätigkeit auch mit anderen Maßnahmen für Mütter in naher Zukunft eine realistische Option wird, ist allerdings auch nicht ausgemacht. Einen Versuch wäre es wert.

\section{Entlastung der aktiven Eltern bei der Familienarbeit}

\section{Ausgangspunkt}

Ohne Berücksichtigung der realexistierenden Familienarbeit bergen alle Formen die Gefahr, nur zu einer Überlastung der Frau (und der wenigen Ausnahmemänner, die die Familienpflichten übernehmen) zu führen. Frauen dürfen neben den ihnen allein auferlegten "Familienpflichten « auch erwerbstätig sein. Ihre zeitliche physische und psychische Belastung steigt ins Unermeßliche, ohne daß ihr persönliches Einkommen diese Steigerung mitmacht. Als wesentlich für die gleichberechtigte Erwerbstätigkeit der Mütter gilt daher die Änderung der familialen Arbeitsteilung und eine grundlegende Strukturveränderung der Erwerbsarbeit. 
Im Diskurs über "Vereinbarkeit « wird zwar stets betont, gerade die familiale Arbeitsteilung lasse sich durch Recht kaum beeinflussen, und durch Arbeitsrecht noch weniger ${ }^{83}$. Dennoch hoffen die meisten Diskutantinnen auf ein stärkeres Engagement der Männer in der Familienarbeit ${ }^{84}$. Ohne eine Realisierung dieser Hoffnung kann eine gerechte Verteilung der gesellschaftlich notwendigen Arbeit zwischen den Geschlechtern nicht erreicht werden. Unbezahlte Haus- und Familienarbeit läßt sich nicht einfach eliminieren, auf daß alle dem leichten Lebenswandel der Männer frönen können. Selbst wenn sie stärker professionalisiert wird als bisher, bleibt notwendigerweise ein »Rest «. Dieser muß gerecht zwischen allen arbeitsfähigen Erwachsenen aufgeteilt werden.

Die bisherigen Erfahrungen mit dem vielbeschworenen Rollenwandel lassen allerdings eine reale Basis für die Hoffnung auf eine Veränderung der Männer vermissen. 1985 enthüllte die sogenannte "Brigitte-Studie $\star^{85}$, daß Frauen, die in einer »Partnerschaft « mit einem Mann leben, spätestens nach der Geburt eines Kindes für den Haushalt alleinzuständig sind ${ }^{86} .1994$ kommt das Statistische Bundesamt in seinem »Zeitreport« zu demselben Ergebnis ${ }^{87}$. Es scheint, als seien Männer weder bereit, den Putzlappen zu schwingen, noch »Beurlaubungen « zur Kinderbetreuung in Anspruch zu nehmen. Auch ausländische Erfahrungen sind alles andere als ermutigend. In Schweden, wo der Erziehungsurlaub mit $80 \%$ des vorherigen Nettogehaltes »entgolten « wird, nehmen Männer nur 5-8\% der insgesamt beanspruchten Elternurlaubstage $^{88}$. In der DDR, in der die Vereinbarkeit von Vollerwerbstätigkeit und Elternschaft kein Problem war, blieb die Hausarbeit trotzdem vorrangig an den Frauen hängen ${ }^{89}$. Ob restriktive Rechtsregeln hieran etwas ändern können, sei dahingestellt. Jedenfalls ist der Vorschlag der österreichischen Frauenministerin, die ehelichen Pflichten um Hausarbeit für Männer anzureichern ${ }^{90}$, innovativ, dasselbe gilt für ein italienisches Volksbegehren, Männer im Rahmen einer Zwangsbeurlaubung von I 2 Monaten zu Pflegediensten heranzuziehen ${ }^{91}$. Selbst eine rechtliche Verpflichtung würde Männer jedoch kaum zum Mitwirken motivieren, da sie nicht vollstreckbar wäre (wie die Änderung des Familienrechts) oder an verfassungsrechtliche Grenzen stoßen würde (wie die Zwangspflege).

Kann die Konsequenz hieraus sein, daß Frauen - oder gar die Frauenbewegung sich stärker der Arbeit am Mann verschreiben? Muß die Realität der Familie, in der es Männern häufig gelingt, mit der Mutterschaft die Alleinverantwortung der Frau für den Haushalt durchzusetzen, in der Politik derart gespiegelt werden, daß mit der Vereinbarkeitsforderung die Frauenbewegung den Mann ins Zentrum des Interesses nimmt? Letztlich wird die Beteiligung der Männer an der Hausarbeit nur in der familiären Beziehung durchsetzbar sein - und das bedeutet, daß Frauen sie einfordern müssen. Daraus folgt nicht die rechtspolitische Konzentration auf den Mann. Die Forderungen der Frauen werden nämlich nur Erfolg haben, wenn sie eine solche Verhandlungsbasis haben - z. B. weil sie Mutterschaft nicht nur bei privater ökonomischer Absicherung leben können, sondern auf der Basis tatsächlich gleichberech-

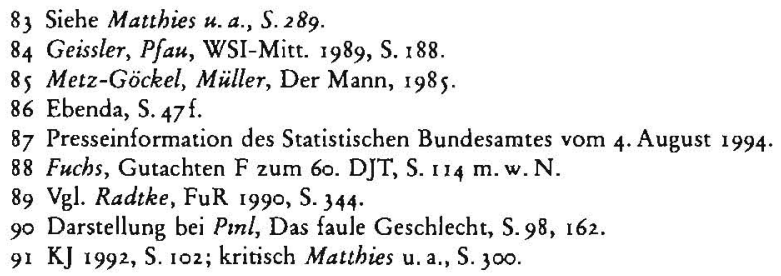


tigter Teilhabe aktiver Eltern - auch alleinstehender - am Erwerbsleben. Dafür wiederum ist die Entlastung aktiver Eltern von Haus- und Erzichungsarbeit durch öffentliche oder öffentlich finanzierte Einrichtungen Voraussetzung. Ein Langzeiteffekt wäre immerhin denkbar. Vielleicht würden Frauen seltener als Hausfrauen zur Verfügung stehen und Beziehungen mit haushalts- und erziehungsfaulen Männern beenden, wenn sie eine Alternative hätten. Vielleicht würden Männer das Zusammenleben mit einer Frau so sehr wünschen, daß sie auch Haus- und Erziehungsarbeit übernähmen.

\section{3. Öfentliche und betriebliche Unterstützung}

Getreu dem Motto »Nur Utopien sind realistisch ${ }^{92}$ seien zunächst die utopischen Forderungen genannt: Vorrangig ist die Sicherung der professionellen Betreuung von Kindern aller Altersgruppen in pädagogisch verantwortbaren Einrichtungen mit ganztägiger Öffnungszeit und Mittagstisch ohne Elternkoch- und Putzverpflichtung. Die Betreuungseinrichtungen dürfen keine Betriebsferien machen; und für Schulkinder muß es Ferienangebote geben. Dies erscheint zwar für Deutschland als Utopie, ist aber in anderen europäischen Ländern die Norm. Auch ausgewogene preiswerte Mahlzeiten für alle Beschäftigten in den Betrieben, preisgünstige (subventionierte) Reinigungsbetriebe für Haushaltswäsche und ggf. Wohnungen wären eine große Erleichterung.

Um auf die Ebene der Realität zurückzukehren: Wartet man auf die Aktivität der Kommunen, so wird die Umsetzung des $\$ 24$ KJHG noch bis zum Jahre 3000 dauern. Ursache hierfür sind nicht nur knappe Mittel, sondern auch die institutionelle Trägheit der personell knapp ausgestatteten Verwaltungen. Die Improvisationsfähigkeit von aktiven Eltern, die Beruf und Familie vereinbaren (müssen), ist demgegenüber erstaunlich. Nach dänischem Vorbild könnte man sie sich zunutze machen. Dort, wo öffentliche Betreuungseinrichtungen nicht oder nicht in erforderlichem Umfang bereitstehen, könnten die kommunalen Gelder statt für den hausfrauengerechten Ausbau der Kindergärten für die Förderung selbstorganisierter Lösungen verwendet werden. Aufgrund des weiblichen Erfindungsreichtums in bezug auf individuelle Lösungen bei der Kinderbetreuung wäre der Betreuungsgrad von Kleinkindern so gewiß schnell auf den europäischen Durchschnitt zu heben. Bisher scheitern selbstverwaltete Einrichtungen, wo nicht an knappen Geldern, häufig an den strengen Anforderungen an die Ausstattung von Kindereinrichtungen. Vom kindergerechten Klo bis zur Größe der Fenster ist alles normiert. Damit werden die Kinder dann häufig in die familiäre Betreuung entlassen, in der die baulichen Anforderungen an KiTas auch nicht erfüllt werden. Hier könnten Bewilligungsrichtlinien flexibler werden.

Auch betriebliche Hilfen sind denkbar. Jenseits der Schaffung oder Subventionierung von Einrichtungen schlagen Matthies u. a. vor, Kinder mit an den Arbeitsplatz zu bringen ${ }^{93}$. Im Notfall mag dies eine Lösung sein, auch wenn bedacht werden muß, daß dies ebenso wie Heimarbeit und Teilzeit zur Mehrfachbelastung der aktiven Eltern führt: Neben der Arbeit im Betrieb sollen sie Kinder betreuen; neben der Heimarbeit zu Hause die Erziehung und Haushaltsführung so gut ausfüllen, daß nichtaktive Eltern nichts merken; neben der vollen Leistung in Teilzeitarbeit im Beruf die volle Leistung einer Vollzeithausfrau/eines Vollzeitshausmannes erbringen. Um betriebliche Hilfen zu intensivieren, könnte - nach dem Vorbild des Arbeitsge- 
setzbuches der DDR - eine betriebliche Verpflichtung zur Bereitstellung von Betreuungsplätzen geschaffen werden, ggf. als Verpflichtung zur Dreieckskooperation von Staat, Unternehmen und Eltern ${ }^{94}$. Dies ist vielleicht gar nicht so utopisch wie es klingt, hält man sich vor Augen, daß die Verpflichtung zur Schaffung von Parkplätzen bei Neuansiedlungen von Betrieben bereits selbstverständlich ist. Muß das Auto der Deutschen liebstes Kind bleiben?

\section{Schattenarbeitsverbältnisse}

Praktisch greifen die meisten aktiven Eltern auf die Hilfeleistungen ungeschützt beschäftigter Frauen zurück. Viele Kinderfrauen und Haushaltshilfen dürften in diese Kategorie fallen. Damit wird ein ungesicherter Arbeitsmarktsektor für Frauen geschaffen - der Gleichstellung am Arbeitsmarkt nicht gerade förderlich. Zweifellos ist gerade bei der Kinderbetreuung eine flexible Notlösung unverzichtbar's. Aber flexible Notlösungen könnten durch öffentliche Subventionierung in sozial gesicherte Beschäftigungsverhältnisse überführt werden. So könnten z. B. "Notmütterdienste" auf der Basis sozialversicherter Beschäftigungsverhältnisse geschaffen werden, für die Eltern einen sozial gestaffelten Preis zahlen, der die Dienste gegenüber der Schwarzarbeit wettbewerbsfähig macht.

\section{Erwerbsarbeitszeit, aktive Elternschaft und der "Kindermalus "der weiblichen Arbeitskraft}

All diese Vorschläge können die Gleichzeitigkeit noch nicht ermöglichen, wenn nicht das Erwerbsarbeitsverhältnis selbst angetastet wird. Erforderlich ist eine effektive Beschränkung der täglichen Erwerbsarbeitszeit auf ein Maß, das die Betreuung eines Kindes und die eigene Reproduktion ermöglicht. Notwendig wäre eine Veränderung der üblichen Arbeitszeit, die so bemessen sein müßte, daß sie die Reproduktion - d.h. Versorgung des eigenen Haushaltes und eines während der eigenen Arbeitszeit betreuten Kindes - ohne gesundheitliche Gefährdung auf Dauer zulas$\operatorname{sen}^{96}$. Eine drastische Verminderung der gesetzlichen Höchstarbeitszeit ist für die Umverteilung der „Familienarbeit« aber nur notwendige, nicht hinreichende Voraussetzung. Es müßte auch gewährleistet werden, daß Männer die Verminderung nicht für flexible Überstundenleistungen, Zweitjobs und zur Verbesserung ihrer Wettbewerbsfähigkeit nutzen. Damit wäre wieder eine utopische Forderung benannt. Wenn die Differenzierung der Arbeitszeiten als Mittel zu mehr "Familiengerechtigkeit « propagiert wird ${ }^{97}$, ist Vorsicht geboten. Jede Differenzierung der Arbeitszeiten läuft Gefahr, die unterschiedlichen Zeitpräferenzen von Frauen und Männern aufgrund der außererwerblichen Arbeitsteilung zu spiegeln. Arbeitszeitdifferenzierung nach Geschlecht führt jedoch - wie jede Differenzierung zwischen männlicher und weiblicher Arbeitskraft - unweigerlich zu schlechteren Marktpositionen für die weibliche Arbeitskraft. Im Kampf gegen geschlechtsspezifische Arbeitsteilung kann Differenzierung der Arbeitszeit nur dann ein Element sein, wenn sie nicht entlang der Geschlechtertrennung erfolgt. Dafür bestehen durchaus Chancen. Frauen, die eine Kinderfrau nur an drei Tagen in der Woche bezahlen können, haben von der Zusammenballung der Arbeitszeit auf die ersten drei Wochentage

94 So Matthres u. a. S. $294 \mathrm{ff}$.

95 Matthies u. a. schlagen betriebliche Listen kurzfristig einsatzbereiter Kinderfrauen vor (S. 294), ohne jedoch zu erläutern, wie deren Arbeitsverhältnisse abgesichert sind.

96 Vgl. Marsh (Fn. 74).

97 So Matthues u. a., S. 292 f., 324 . 
ebensoviel wie Männer, die einem Zweitjob oder einem Hobby oder der Landwirtschaft frönen wollen. Auch hier besteht immer noch die Gefahr, daß Männer sich weiterbilden oder in den Nebenjobs beruflich wertvolle Kontakte aufbauen, während Frauen »nur « eine außerfamiliäre Karriere aufbauen. In dieser wären sie dann aber nicht schon durch das Arbeitszeitarrangement als "Familienfrau « diskriminiert.

Zur Kompatibilität von Erwerbsarbeit und aktiver Elternschaft gehört auch ein effektiver Mutterschutz ohne zusätzliche Kosten für Arbeitgeber im Fall der Schwangerschaft. Die ${ }_{14}$ Wochen Schutzfristen bei Einkommenssicherung sind zu kurz. Wenn man zum 26wöchigen Mutterschutz der DDR bei voller Einkommenssicherung zurückkehren könnte, wäre der Erzichungsurlaub für viele Mütter sicherlich weniger attraktiv. Jedenfalls müßten sie nicht Erziehungsurlaub nehmen, um die rein körperliche Erholung nach der Geburt zu erreichen, die zur Aufnahme einer Erwerbstätigkeit nötig ist. Die Befreiung der Frau vom Kinderrisiko setzte allerdings zweierlei voraus:

- Übernahme aller Kosten (d. h. der Lohnfortzahlung und ggf. zusätzlicher Kosten, die die Einstellung einer Ersatzkraft verursacht) durch öffentliche Kassen.

- Paralleler Vaterschaftsschutz durch ein Beschäftigungsverbot in gleicher Länge ${ }^{98}$ letzteres sicher wieder im Bereich der Utopien, aber trotzdem eine Möglichkeit, wie beide Eltern unmittelbar nach der Geburt die gleichberechtigte Teilung der Elternschaft einüben könnten.

Der Erhalt des Erziehungsurlaubs in der gegenwärtigen Form läßt sich mit der Forderung der Entlastung der Frauen vom Kinderrisiko sicherlich nicht vereinbaren. Wie die gleichgewichtige Beteiligung der Väter an dieser Einrichtung erreicht werden kann, ist nämlich völlig offen. Untauglich wäre sicherlich der Versuch, (Ehe-)Paaren den dreijährigen Erziehungsurlaub nur zuzugestehen, wenn beide jeweils die Hälfte nehmen ${ }^{99}$. Männer könnten - und würden - ihre Hälfte straflos verweigern. Wie unrealistisch die Hoffnung auf die Beteiligung der Väter ist, zeigen die o. g. Ergebnisse der Beanspruchung des Kinderkrankengeldes: Wenn Männer zehn Tage im Jahr lieber verfallen lassen als zuzugeben, daß sie sich für ihre Kinder verantwortlich fühlen, kann nicht davon ausgegangen werden, daß sie ein Jahr Beurlaubung in Anspruch nehmen. Fordert man weiterhin, der Partner einer »Erziehungsurlauberin « müsse sich bereits bei Antritt des Urlaubs verpflichten, seinen Urlaub im Anschluß zu nehmen, könnte dies durch Trennung/Scheidung problemlos umgangen werden. Möglicherweise könnte die Verpflichtung, die Erziehungsurlaubsphasen in "Portionen " von maximal sechs Monaten aufzuteilen, die Unterbrechungsdauer bei Frauen und damit die faktischen Nachteile vermindern.

Denkbar ist aber auch, daß sie mangels Kinderbetreuungseinrichtungen und unter dem moralischen Druck in Richtung höchstpersönlicher Kinderbetreuung ungeschützte "Beurlaubung in Anspruch und damit noch größere Risiken auf sich nehmen. Solche Folgen könnte jede Zurückdrängung der extensiven Förderung des Vierphasenmodells haben. Möglicherweise ist dies der politischen Durchsetzbarkeit abträglich. Will man aber ernsthaft den Kindermalus abschaffen oder zumindest verringern, der gegenwärtig über jeder Arbeitnehmerin im gebärfähigen Alter schwebt, muß die Unterbrechung der Erwerbsarbeit außerhalb des Mutterschutzes vermieden werden. Dann erst könnten Unternehmen davon ausgehen, durch die Beschäftigung von Frauen auf Positionen, für die sie auch Männer gewinnen könnten, keine Wettbewerbsnachteile zu erleiden. 
Trotz solcher Idealbedingungen würde sich die gleichmäßige Beschäftigung von Familienfrauen auf allen Positionen sicherlich nicht im Selbstlauf durchsetzen. Zu tief sitzen die Vorurteile über die wahre Bestimmung der Mutter als höchstpersönliche Kinderbetreuerin, als daß Personalchefs davon überzeugt wären, Frauen würden auf diese Bestimmung verzichten. Zu stark ist die Gewöhnung an den Arbeitsmarkt der Verantwortungslosen - d. h. an die unbeschränkte Verfügbarkeit von Arbeitskräften, die die Energie der hinter ihnen stehenden Hausfrau mit in den Erwerbsprozeß einbringen, als daß Unternehmen ohne sanften Druck Arbeitskräfte akzeptieren, die dies nicht bieten. Wirksame Frauenquoten könnten dazu beitragen, diese Gewöhnungen zu durchbrechen. Wenn sie gewährleisten, daß sich der Anteil von Frauen in allen Wirtschaftsbereichen signifikant erhöht, wird sich zeigen, daß die Auflockerung des Grau der Vorstandsetagen nicht zum Ruin der deutschen Wirtschaft führt; wird erkennbar werden, daß Selbstverantwortung für Reproduktion nicht qualifikationsmindernd sein muß; wird bewiesen werden, daß auch ohne kostenlose Arbeitskraft im Hintergrund Erwerbsarbeit effektiv geleistet werden kann.

Häufig wird die »Vereinbarkeit« als notwendige Rahmenbedingung für die Durchsetzung von Frauenfördermaßnahmen bezeichnet ${ }^{1 \infty}$, und diese Einschätzung ist sicherlich zutreffend: Ohne Vereinbarkeitsregeln werden von Quoten nur kinderlose Frauen profitieren. Dementsprechend enthalten alle neueren Landesgleichstellungsgesetze Vereinbarkeitsregelungen ${ }^{101}$. Zu wenig ist bisher daneben beachtet worden, daß die Vereinbarkeit von Erwerbstätigkeit und Familie nicht nur notwendige Ergänzung der Quote ist, sondern daß ohne verbindliche Frauenquoten die Vision der Gleichzeitigkeit von Erwerbstätigkeit und elterlicher Verantwortung niemals Wirklichkeit werden wird, weil Menschen mit »Familienpflichten« - d. h. Mütter - im Erwerbsleben niemals selbstverständlich werden.

\section{Abschied von der "Vereinbarkeit im bekannten Sinne}

Die ökonomische Eigenständigkeit von Müttern, die nur durch möglichst durchgehende Integration ins Erwerbsleben erreicht werden kann, gibt Frauen die Chance, Forderungen an die Gestaltung der familiären Arbeitsteilung auch durchzusetzen und ermöglicht damit, daß Väter in relevantem Ausmaß in die Verantwortung genommen werden. Dies kann aber nur durch Abkehr vom Vierphasenmodell und Hinwendung zum Aufbau einer Infrastruktur, die durchgehende Erwerbstätigkeit auch für Alleinerziehende ermöglicht, erfolgen. Die Durchsetzung der Gleichstellung im Erwerbsleben fordert deshalb auch die Abkehr vom Leitbild der Hausfrauenehe im Arbeitsrecht. Die weitere Ausdifferenzierung von familienbedingten Beurlaubungen und familienbedingter Teilzeitarbeit ohne Existenzsicherung für aktive Eltern und ihre Kinder - die bislang alle Parteien unter dem Stichwort Vereinbarkeit propagieren - führt in eine Sackgasse. Ergebnis wäre eine Vertiefung der geschlechtsspezifischen Spaltung des Arbeitsmarktes und auf lange Sicht eine Verarmung von Müttern und Kindern bei fortbestehender Verantwortungslosigkeit der Väter.

100 So Battis, Eisenhard, ZRP 1994, S. 2 s.

IOI Die beiden älteren Gesetze aus Nordrhein-Westfalen und dem Saarland werden durch Frauenforderkonzepte ergänzt, die ähnliche Maßnahmen enthalten. 
Unsere Verfassung gewährt das Grundrecht auf Petition (Art. 17 GG). Es vermittelt den Anspruch auf sachliche Prüfung einer Frage oder Beschwerde ohne eine bestimmte Rechtsfolge vorzugeben, ist also ein rein formelles Recht. Aber auch die Erledigung formeller Ansprüche läßt manchmal Erkenntnisse aufblitzen, die verfassungspolitisch aufhorchen lassen und verfassungsrechtlich zu denken geben. Dies ist der Fall bei einer Beschlußempfehlung des Petitionsausschusses des Deutschen Bundestages (Pet 4-12-07-301-55712), die aufgrund einer am 5. Oktober 1993 erhobenen Petition eines bekannten Kölner Steueranwaltes erging.

Der Petent hatte geltend gemacht, sein Grundrecht auf Meinungsfreiheit sei durch den Präsidenten des Bundesfinanzhofs, Prof. Dr. Franz Klein, sowie durch den Vizepräsidenten des gleichen Gerichts, Prof. Dr. Klaus Offerhaus, verletzt worden; beide hätten ihre Dienststellung dazu mißbraucht, seinen Ausschluß als ständigen Mitarbeiter der vom Stollfuß-Verlag herausgegebenen Deutschen Steuer-Zeitung (DStZ) zu veranlassen. Der Präsident des Bundesfinanzhofs habe unter aktiver Unterstützung seines Vizepräsidenten den Verlagsleiter des Stollfuß-Verlages vor die Wahl gestellt, zwischen ihm und dem Petenten als ständigem Mitarbeiter der DStZ zu wählen. Der Verlagsleiter habe sich gegen den Petenten und für den Präsidenten des Bundesfinanzhofs als ständigen Mitarbeiter entschieden, weil der Stollfuß-Verlag, der die amtliche Sammlung der BFH-Entscheidungen sowie die BFH-Entscheidungssammlung "Höchstrichterliche Finanzrechtsprechung « herausgebe, vom Bundesfinanzhof wirtschaftlich abhängig sei. Klein als Präsident des Bundesfinanzhofs habe damit seine dienstliche Machtstellung ausgenutzt, um die wissenschaftliche Mitwirkung des Petenten an der DStZ zu unterbinden, und habe damit dessen Grundrecht auf Meinungsfreiheit verletzt.

Der Petitionsausschuß kommt unter Berufung auf die von ihm eingeholte Stellungnahme des Bundesjustizministeriums zum Ergebnis, die Mitherausgabe einer Fachzeitschrift stelle keine dienstliche, sondern eine rein privatwissenschaftliche Betätigung des Präsidenten des Bundesfinanzhofs dar, innerhalb derer eine Grundrechtsbindung nicht bestehe. Es stehe dem Präsidenten frei zu entscheiden, mit wem er außerdienstlich zusammenarbeiten wolle; seine Erklärung gegenüber dem StollfußVerlag, er lehne es ab, zusammen mit dem Petenten die Funktion eines ständigen Mitarbeiters der DSt $Z$ wahrzunehmen, vermöge eine Grundrechtsverletzung des Petenten nicht zu bewirken. Mit dieser Argumentation kommt der Petitionsausschuß zur Beschlußempfehlung, das Petitionsverfahren abzuschließen.

Dies alles wäre an sich keiner besonderen Beachtung wert, denn es ist eine verfassungsrechtliche Binsenwahrheit, daß es - zumindest prinzipiell - eine Grundrechtsbindung im Privatrechtsverkehr nicht gibt und daß sich ein Verleger im Rahmen der Vertragsfreiheit für einen bestimmten Mitarbeiter (konkret: Klein als Präsidenten des Bundesfinanzhofs) und gegen einen anderen (konkret: den Petenten) entscheiden kann. Von Interesse ist insofern auch kaum das mit dürren Worten abgeleitete Ergebnis dér Beschlußempfehlung des Petititionsausschusses als vielmehr der der Petition zugrundeliegende Sachverhalt, wird er in seinem vollständigen Zusammenhang erfaßt. Denn: wenn in der Beschlußfassung gesagt wird, "zwischen dem Präsidenten des Bundesfinanzhofs einerseits und dem Petenten andererseits» bestün- 